\title{
The structure of amorphous calcium sulfate and its role in the nucleation pathway and final mesostructure of $\mathrm{CaSO}_{4}$ phases
}

\author{
TOMASZ MACIEJ STAWSKI ${ }^{1}$ AND ALEXANDER E.S. \\ VAN DRIESSCHE ${ }^{2}$ \\ ${ }^{1}$ Federal Institute for Materials Research and Testing (BAM) \\ ${ }^{2}$ ISTerre, Univ. Grenoble-Alpes, CNRS \\ Presenting Author: tomasz.stawski@bam.de
}

In recent years, we have come to appreciate the astounding intricacy of the formation process of minerals from ions in aqueous solutions. In this context, a number of studies have already revealed that nucleation in the $\mathrm{CaSO}_{4}-\mathrm{H}_{2} \mathrm{O}$ system is non-classical, where the formation of the different crystalline phases involves several steps including a common amorphous precursor. In this contribution a holistic view of the formation mechanism of gypsum and bassanite from solution will be presented.

In short, our in situ and time-resolved scattering data demonstrate that calcium sulfate precipitation starts with the formation and aggregation of well-defined sub- $3 \mathrm{~nm}$ primary species. These species constitute building "bricks" of an amorphous precursor phase. We characterised the "bricks" by combining information obtained at different length-scales accessible at the mesoscale (from small-angle scattering) and at the atomic-length-scale (wide-angle scattering and high-energy diffraction). From these scattering data we derived pair distribution functions of the clusters and restricted their external shapes and dimensions. This allowed us to propose a structure of the primary species and to explore their dynamic properties with unbiased MD simulations using polarizable force fields. The formation of the amorphous phase involves the aggregation of these small primary species into larger disordered aggregates exhibiting "brick-in-the-wall" structure. The actual crystallisation occurs by the restructuring and coalescence of the "bricks" into a given calcium sulfate phase depending on the thermodynamic conditions of the solution. Importantly, these rearrangement processes by no means continue until a (nearly)perfect homogeneous single crystal is obtained. Instead they come to a stop or at least significantly slow down. Such a process thus yields a final imperfect mesocrystal, composed of smaller domains rather than a continuous crystal structure, within which the domains are separated by an amorphous calcium sulfate phase. 